\title{
Analysis and Comparison of P2P Search Methods
}

\author{
Dimitrios Tsoumakos \\ Department of Computer Science \\ University of Maryland \\ College Park, MD 20742, USA \\ dtsouma@cs.umd.edu
}

\author{
Nick Roussopoulos \\ Department of Computer Science \\ University of Maryland \\ College Park, MD, 20742, USA \\ nick@cs.umd.edu
}

CS-TR-4539, UMIACS-TR-2003-107

\begin{abstract}
The popularity and bandwidth consumption attributed to current Peer-to-Peer file-sharing applications makes the operation of these distributed systems very important for the Internet community. Efficient object discovery is the first step towards the realization of distributed resource-sharing. In this work, we present a detailed overview of recent and existing search methods for unstructured Peer-to-Peer networks. We analyze the performance of the algorithms relative to various metrics, giving emphasis on the success rate, bandwidth-efficiency and adaptation to dynamic network conditions. Simulation results are used to empirically evaluate the behavior of nine representative schemes under a variety of different environments.
\end{abstract}

\section{INTRODUCTION}

Peer-to-Peer (hence P2P) computing represents the notion of sharing resources available at the edges of the Internet [1]. Its initial success (e.g., Napster [2]), boosted the interest in this new approach. A large number of systems and architectures that utilize P2P technology have emerged since ( [3]-[6], etc.). The P2P paradigm dictates a fully-distributed, cooperative network design, where nodes collectively form a system without any supervision. Its advantages (although application-dependent in many cases) include robustness in failures, extensive resource-sharing, self-organization, load balancing, data persistence, anonymity, etc.

According to a very conservative estimate [1], there exist more than $10 \times 10^{9} \mathrm{MHz}$ of CPU power and 10,000 TB of storage not utilized at the edges of the Internet. According to a different report [7], bandwidth consumption attributed to popular file-sharing applications amounts to a considerable fraction (up to 60\%) of the total Internet traffic. These two reports identify two different problems: First, there is a vast amount of "untapped" potential around the Internet. On the other hand, current resource-sharing applications waste huge amounts of bandwidth. P2P technology can play a key role in our efforts to tackle 
both issues. In all cases, the first step involves the efficient discovery of the various resource locations inside a network.

Today, the most popular P2P applications operate on unstructured networks. In these networks, peers connect in an ad-hoc fashion, the location of the documents is not controlled by the system and no guarantees for the success or the complexity of a search are offered. Search methods can be categorized as either blind or informed, according to whether peers utilize information to locate resources. In a blind search, nodes hold no information that relates to document locations, while in informed methods, there exists a centralized or distributed directory service that assists in object discovery.

In this work, we describe a variety of proposed search algorithms for unstructured P2P networks. A search process includes aspects such as the query-forwarding method, the set of nodes that receive queryrelated messages, the form of these messages, local processing, stored indices and their maintenance, etc. This paper describes current approaches from both (blind and informed) categories and analyzes their performance. We focus on the behavior of these algorithms for each of the following metrics:

- Efficiency in object discovery (accuracy and number of hits)

- Bandwidth consumption

- Adaptation to changing topologies and workloads

The first metric measures search accuracy and the number of discovered objects per request (this is very important for many applications, as it makes object retrieval very scalable). Minimizing message production always represents a high-priority goal for all distributed systems. Finally, it is important that any search algorithm adapts to changing conditions, since in most P2P networks users frequently enter and leave the system, as well as update their collections.

To evaluate our analysis, we simulate nine of the described methods and present a direct quantitative comparison of their performance. We identify the relative advantages and disadvantages of each method as well as the conditions under which they can be most or least effective. We believe this is an important contribution that can provide a better understanding of the various search mechanisms and assist in choosing an algorithm that best fits a particular application.

The rest of this paper is organized as follows: Section II presents the related work. In Section III we classify and describe many current search techniques, while in Section IV we present the simulation results. Finally, Section V contains our conclusions.

\section{RELATED WORK}

Peer-to-Peer networks have been studied a lot in the last few years. A large amount of information for P2P computing with taxonomies, definitions, current trends, applications and related companies can be obtained at [8], [9], as well as individual sources (e.g., [5], [6]). P2P computing is also described in [10], with basic terminology, taxonomies and description of some systems. A brief summarization of Gnutella [3] and Napster [2] search schemes, together with approaches for structured networks are also included. 
Gnutella and Napster are the focus of two measurement studies; Ref. [11] attempts a detailed characterization of the participating end-hosts, while [12] measures the locality of stored and transferred documents. In [13], a traffic measurement for three popular P2P networks is being conducted at the border routers of a large ISP. Extensive results for traffic attributed to HTTP, Akamai and P2P systems are also presented in [14].

Quantitative comparisons between the search methods in [15], [16] and the original Gnutella algorithm are presented in these two papers. Their main comparison metric is bandwidth consumption. The work in [17] presents a thorough comparison between the APS algorithm and the search schemes introduced in [16], [18] on a variety of metrics.

Our work focuses exclusively on proposed search methods for unstructured P2P networks and provides a direct experimental comparison of these algorithms under different environments.

\section{P2P SEARCH AlgorithMS}

\section{A. Our Framework}

First, we briefly describe our system model for search in structure-less P2P networks. Each peer retains a local collection of documents, while it makes requests for those it wishes to obtain. The documents are stored at various nodes across the network. Peers and documents (or objects) are assumed to have unique identifiers, with object IDs used to specify the query target. Objects are assumed to be of varying popularity, which affects the respective number of replicas and received requests. Objects are distributed over the network according to the replication distribution, which dictates the number and identity of objects stored at each node. Each peer makes requests according to a query distribution, which controls how many requests are made for each object (e.g., popular objects get many more requests than unpopular ones).

Search algorithms cannot in any way dictate object placement and replication in the system. Nodes that are directly linked in the overlay are neighbors. Peers obtain only a local knowledge of the network (e.g., are only aware of their neighbors). We also assume that they can keep soft state (i.e., information that is erased after a short amount of time) for each query they process. Each search is assigned an identifier, which, together with the soft state, enables peers to make the distinction between new queries and duplicate ones.

A search is successful if it discovers at least one replica of the requested object. The ratio of successful to total searches made is called the success rate (or accuracy). A search can result to multiple discoveries (or hits), which are replicas of the same object stored at distinct nodes. A global TTL parameter represents the maximum hop-distance a query can reach before it gets discarded.

\section{B. Search Taxonomy}

There are two possible strategies used to search for an object: Search in a blind fashion, trying to propagate the query to a sufficient number of nodes in order to satisfy the request; or utilize information 
about document locations and thus perform an informed search. The semantics of the used information range from simple forwarding hints to exact object locations. The placement of this information can also vary: In centralized approaches (e.g., [2]), a central directory known to all peers exists. Distributed approaches can also be sub-divided into pure and hybrid. In pure approaches (e.g., [15], [17], [19]), all participating peers maintain some portion of the information. Other algorithms (e.g., [20]) operate on hybrid $\mathrm{P} 2 \mathrm{P}$ architectures, where certain nodes assume the role of a super-peer and the rest become leafnodes. Each super-peer acts as a proxy for its leaf-nodes by indexing all their documents and serving their requests.

The semantics of the stored indices in informed approaches can be used for another categorization. Indices might relate to exact object locations (e.g., [21]), probability of discovery through a link (e.g., [17]), number of objects through a link (e.g., [19]), or even other metrics (e.g., [22]).

Finally, we can categorize search schemes according to the query forwarding method into flood-based (utilizing the standard flooding scheme or one of its variations) and non flood-based (e.g., [16], [23]).

\section{Blind Search Methods}

GNUTELLA [3]: The original Gnutella algorithm uses flooding (BFS traversal of the underlying graph) for object discovery, contacting all accessible nodes within $T T L$ hops. Its basic characteristics are its simplicity and the huge overhead it produces by contacting many nodes.

Modified-BFS [15]: In this variation of the flooding scheme, peers randomly choose only a ratio of their neighbors to forward the query to. This algorithm certainly reduces the average message production compared to the previous method, but still contacts a large number of peers.

Iterative Deepening: Two similar approaches that use consecutive BFS searches at increasing depths are described in [16], [24]. These algorithms achieve best results when the search termination condition relates to a user-defined number of hits and it is possible that a "small-range" flood will satisfy the query. In a different case, they produce even bigger loads than the standard flooding mechanism.

Random Walks [16]: In Random Walks, the requesting node sends out $k$ query messages to an equal number of randomly chosen neighbors. Each of these messages follows its own path, having intermediate nodes forward it to a randomly chosen neighbor at each step. These queries are also known as walkers. A walker terminates either with a success or a failure. Failure can be determined by two different methods: The $T T L$-based method and the checking method, where walkers periodically contact the query source asking whether the termination conditions have been satisfied. The algorithm's most important advantage is the significant message reduction it achieves, since it produces $k \times T T L$ messages in the worst case. Results in [16], [17] show that messages are reduced by more than an order of magnitude compared to the standard flooding scheme. It also achieves some kind of local "load balancing", since no nodes are favored in the forwarding process over others. The most serious disadvantage of this algorithm is its highly variable performance. Success rates and number of hits vary greatly depending on network topology and the random choices made. Another drawback of this method is its inability to adapt to different query 
loads, since queries for popular and unpopular objects are treated in the exact same manner.

Recently, two new search protocols which operate on hybrid topologies made their appearance:

GUESS [18]: This algorithm builds upon the notion of Ultrapeers [25]. Each ultrapeer is connected to other ultrapeers and to a set of leaf-nodes (peers shielded from other nodes), acting as their proxy. A search is conducted by iteratively contacting different ultrapeers (not necessarily neighboring ones) and having them ask all their leaf-nodes, until a number of objects are found. The order with which ultrapeers are chosen is not specified.

Gnutella2 [20]: In Gnutella2 (G2), when a super-peer (or $h u b$ ) receives a query from a leaf, it forwards it to its relevant leaves and also to its neighboring hubs. These hubs process the query locally and forward it to their relevant leaves. No other nodes are visited with this algorithm. Neighboring hubs regularly exchange local repository tables to filter out unnecessary traffic.

Although the details of these protocols are still formulating, we observe they rely on a dynamic hierarchical structure of the network. They present similar solutions for reducing the effects of flooding by utilizing the structure of hybrid networks. The number of leaf-nodes per super-peer must be kept high, even after node arrivals/departures. This is the most important condition in order to reduce message forwarding and increase the number of discovered objects.

\section{Informed Search Methods}

Intelligent-BFS [15]: This is an informed version of modified-BFS. Nodes store query-neighborID tuples for recently answered requests from (or through) their neighbors in order to rank them. First, a peer identifies all queries similar to the current one, according to a query similarity metric; it then choses to forward to a set number of its neighbors that have returned the most results for these queries. If a hit occurs, the query takes the reverse path to the requester and updates local indices. This approach focuses more on object discovery than message reduction. At the cost of an increased message production compared to modified-BFS (because of the update process), the algorithm increases the number of hits. It achieves high accuracy, enables knowledge sharing and induces no overhead during node arrivals/departures. On the other hand, its message production is very large and only increases with time as knowledge is spread over the nodes. It shows no easy adaptation to object deletions or peer departures, because the algorithm does not utilize negative feedback and forwarding is based on ranking. Finally, its accuracy depends highly on the assumption that nodes specialize in certain documents.

APS [17]: In APS, each node keeps a local index consisting of one entry for each object it has requested per neighbor. The value of this entry reflects the relative probability of this node's neighbor to be chosen as the next hop in a future request for the specific object. Searching is based on the deployment of $k$ walkers and probabilistic forwarding. Each intermediate node forwards the query to one of its neighbors with probability given by its local index. Index values are updated using feedback from the walkers. If a walker succeeds (fails), the relative probabilities of the nodes on the walker's path are increased (decreased). The update procedure takes the reverse path back to the requester and can take place either 
after a walker miss (optimistic update approach), or after a hit (pessimistic update approach). APS employs an indexing scheme that refines its knowledge as queries are made. Every node on the deployed walkers updates its indices according to search results, so peers eventually share and adjust this knowledge with time. Walkers are directed towards objects or redirected if a miss occurs. APS is also very bandwidthefficient (achieving very similar levels with Random Walks) and induces zero overhead over the network at join/leave/update operations. The s-APS modification adaptively switches between the optimistic and pessimistic approaches to minimize the amount of updates along the reverse paths. Since APS gains from knowledge build-up, these advantages are mainly seen when many different peers contribute with big workloads. Moreover, both APS and Random Walks have $k$ as an upper bound in their hits per search.

Local Indices (LI) [24]: Each node indexes the objects stored at all nodes within a certain radius $r$ and can answer queries on behalf of all of them. A search is performed in a BFS-like manner, but only nodes accessible from the requester at certain depths process the query. To minimize the overhead, the hop-distance between two consecutive depths must be $2 r+1$. This approach resembles the two search schemes for hybrid networks. The method's accuracy and hits are very high, since each contacted node indexes a whole neighborhood. On the other hand, message production is comparable to the flooding scheme, even if the processing time is smaller because many nodes just forward the query. The scheme also requires a flood with $T T L=r$ whenever a node joins/leaves the network or updates its local repository, so the overhead becomes even larger for dynamic environments.

GIA [22]: In GIA, requesting nodes deploy biased walkers in order to discover various objects. Each peer chooses to forward the query to the neighbor with the highest announced capacity. This is a userdefined metric that reflects the processing power of a node inside the system. Moreover, the protocol requires that each peer indexes the documents of its neighbors. This scheme also provides for a topology adaptation algorithm which runs continuously. Its goal is to re-configure the overlay connectivity such that each node is connected to a number of peers proportional to its capacity. What this achieves is that the biased walkers are in fact directed to the highly connected neighbors and, probabilistically, to those with the highest number of indexed objects. Finally, the scheme provides a flow-control mechanism which allows peers to control the rate at which they can accept and process requests from their neighbors. Once the topology has been set, we expect GIA to perform very bandwidth-efficient searches with several hits. On the other hand, the adaptation algorithm plus the indexing of the neighbors' repositories increase the responsibilities of each peer as well as the communication overhead. Another issue is how fast can the algorithm work for joining peers and at what cost for their neighborhood.

Routing Indices (RI) [19]: Documents are assumed to fall into a number of thematic categories. Each node stores an approximate number of documents from every category that can be retrieved through each outgoing link (i.e., not only from that neighbor but from all nodes accessible from it). The query termination condition always relates to a minimum number of hits. The forwarding process is similar to DFS: A node that cannot satisfy the query stop condition with its local repository will forward it to the neighbor with the highest "goodness" value. Three different functions which rank the out-links according 
to the expected number of documents discovered through them are also defined. The algorithm backtracks if more results are needed. This is another keyword-search approach which trades index maintenance overhead for increased accuracy. While a search is very bandwidth-efficient, RIs require flooding in order to be created and updated, so the method is not suitable for highly dynamic networks. Moreover, stored indices can be inaccurate due to thematic correlations, over- or under-counts in document partitioning and network cycles.

In [23], each node holds $d$ bloom filters for each neighbor. A filter at depth $i$ summarizes documents that can be found $i$ hops away through that specific link. Nodes forward queries to the neighbor whose smaller depth bloom filter matches a hashed representation of the object ID. After a certain number of steps, if the search is unsuccessful, it is handled by a deterministic algorithm instead of backtracking. The scheme's expectation is to find only one replica of the object with high probability. Index maintenance requires flooding messages initiated from nodes that arrive or update their collections.

Distributed Resource Location Protocol (DRLP) [26]: Nodes with no information about the location of a document forward the query to each of their neighbors with a certain probability. If an object is found, the query takes the reverse path to the requester, storing the document location at those nodes. In subsequent requests, nodes with indexed location information directly contact the specific node. If that node does not currently obtain the document, it just initiates a new search as described before. This algorithm initially spends many messages to find the locations of an object. In subsequent requests, it might take only one message to discover it. Obviously, a small message production is achieved only with a large workload that enables the initial cost to be amortized over many searches. In rapidly changing networks, this approach fails and more nodes have to perform blind search. This also affects the number of hits: If many blind searches are made, then many results are found; if many direct queries take place, then only one replica is retrieved.

Gnutella with Shortcuts (GS) [21]: In this work, the authors propose the addition of shortcuts (i.e., direct links to peers that have recently proved useful in answering queries) to a Gnutella-like overlay. The original flooding mechanism is initially used to locate documents. Peers that provide answers are indexed by the requesters, following the assumption that they could provide answers to more requests. When a new query is made, nodes first forward it to their shortcuts (ranked in a descending order of usefulness - usually the success rates). If all shortcuts fail, the standard flooding scheme is again used to locate the object. This approach resembles the DRLP scheme but stores more than one pointer and keeps statistics on them. For semantically related queries, we expect it to quickly identify relevant peers and mostly use the shortcuts for object location. Moreover, we anticipate a very high success rate since the fall-back mechanism is flooding. On the other hand, if peers make many unrelated queries or they do not store relevant content, it is possible that the shortcuts will fail, which in turn means that the system pays the price of a full-scale flooding. The same is true when objects are removed or peers depart frequently. 


\section{E. New Approaches}

Structured P2P systems (also known as Distributed Hash Tables - DHTs) base all operations on an "overlay" network, which handles file and replica placement and guarantees bounded number of steps and reliable storage. Recently, there has been an effort to combine the advantages of both DHTs and unstructured systems. In [27], an immediate neighborhood area is defined for each peer. Object placement inside these overlapping areas is performed in a DHT-like fashion. Searches use the standard flooding mechanism except that only certain areas are probed. In [28], peers are grouped into possession rules, according to whether they contain a specific item or not. Nodes search inside one possession rule in a blind fashion. The possession rule is chosen by a greedy mechanism according to past query results.

\section{Simulation Results}

\section{A. Simulation Model and Algorithm Implementations}

In this section we present results for nine of the described methods: (G2, Random Walks, Modified-BFS, Intelligent-BFS, Local Indices, s-APS, DRLP, GS and GIA). The simulated methods are representative blind and informed schemes, both flood and non flood-based, with or without user-initiated index updates (that is, updates triggered strictly by the search process). We briefly summarize our simulation model here.

We mainly use random topology graphs of 10,000 nodes and varying out-degrees generated by GT-ITM [29] to simulate the P2P overlay structure. We assume a pure P2P model, where all peers equally make and forward requests. We also test the performance of the algorithms using the popular power-law model with graphs generated by Inet 3.0 [30].

Queries are made for 100 objects, with object 1 being the most popular and object 100 the least. The small number of objects enables a good observation of the effect that popularity has over the performance of a search. A zipfian distribution with parameter $a=0.82$ is used to model both query and replication distributions and achieve workloads similar to [12]: The top-10\% of the objects account for about $50 \%$ of the total number of stored objects and receive about $50 \%$ of all requests. The replication ratios range from $11 \%$ to $0.25 \%$ (least popular object). With this distribution, there exist around 8,500 replicas of the 100 objects inside our network (each peer holds less than one object on average). Requester nodes are randomly chosen and represent about $20 \%$ of the total number of nodes. Each requester makes about 1,500 queries over a time period. We do not allow extra replicas to be stored (i.e., we only consider the search phase, not object retrieval). The $T T L$ parameter was set to 5 , since larger values produced very similar results. Results were also collected for 20,000 objects with 5,000 requester nodes (each making 2,000 queries).

To simulate dynamic network behavior, we insert "on-line" nodes and remove active ones with varying frequency. The objects are also re-distributed to model file insertions and deletions. We always keep approximately $80 \%$ of the network nodes active, while arriving nodes start functioning without utilizing any (possibly built) prior knowledge. Object re-location always follows the initial distribution parameters. 
The Intelligent-BFS method was modified to allow for object-ID requests. Index values at peers now represent the number of replies for an object through each neighbor and nodes choose the neighbors with the highest index values when forwarding a query. For Modified-BFS's, DRLP's and Intelligent-BFS's flood-based search, nodes choose an equal number of neighbors to forward a query in order to make direct comparisons. For $G 2 / G U E S S$, peers randomly choose $k$ neighbors to forward the query to. The chosen nodes forward the query to all their neighbors. By modifying the value for $k$ we can simulate the operation of both $G 2$ (with $k$ always larger than the average node degree) and GUESS. In our simulations, G2/GUESS operate on a pure (instead of a hybrid) model in order to achieve uniformity in our results. Moreover, they both function in a blind manner, so no cache or repository table exchange takes place. We name this approach $H G 2$ (Hybrid G2/Guess). For our $L I$ implementation, nodes index the objects of their neighbors $(r=1)$. To ensure that the search is equivalent to a flood with $T T L=5$, only peers at depths 1 and 4 process the query. We also ensure that no object from the same peer is being discovered multiple times. Again for the purpose of reporting comparable results, our GIA implementation does not use the overlay adaptation process. $k$ random walkers are independently deployed, and each peer forwards to the neighbor with the highest out-degree (similar to the method described in [31]). Peers index the documents of their neighbors. For our GS implementation, we use 5 shortcuts and rank them by their success rates.

\section{B. Basic performance comparison}

In our first set of experiments, we use a Gnutella-type graph (average degree $d=4$ ) to compare the nine methods over 5 different environments: A static one, one with low/high object relocation frequency and one with low/high peer departure frequency. In the two low-frequency scenarios, relocation and departures/arrivals occur about 300 times per run, while in the high-frequency ones they occur 10 times more often. DRLP and Int/Mod-BFS forward to 3 neighbors, while $k=7$ for $s$-APS, GIA, HG2 and Random Walks. Figures 1 and 2 present the results.

Blind methods show a fairly stable performance between the static and dynamic settings, since the dynamic operations do not interfere with the forwarding scheme. Flood-based schemes discover many objects at a higher cost. Nevertheless, only $L I$ and $G S$ with the pure-flooding scheme achieve very high accuracy. This happens because of the small out-degree of our network. We also notice that blind and flood-based techniques do not get affected by object relocation, but only by peer joins/leaves. While our relocation process does not substantially alter anything in those algorithms' operation, peer arrivals/departures alter the topology and the amount of available resources.

Mod/Int-BFS show relatively high accuracy and return many hits. Their performance is very similar, with the informed method showing marginally better results. For environments resembling this setup, the modified method will be preferred, since its performance is equally high and it is much simpler. We expect the informed method to perform better in richer or more specialized environments (like the one described in [15]), mainly in the number of hits.

Random Walks displays low accuracy $(<34 \%)$ and finds less than 0.5 objects on average. Its bandwidth 

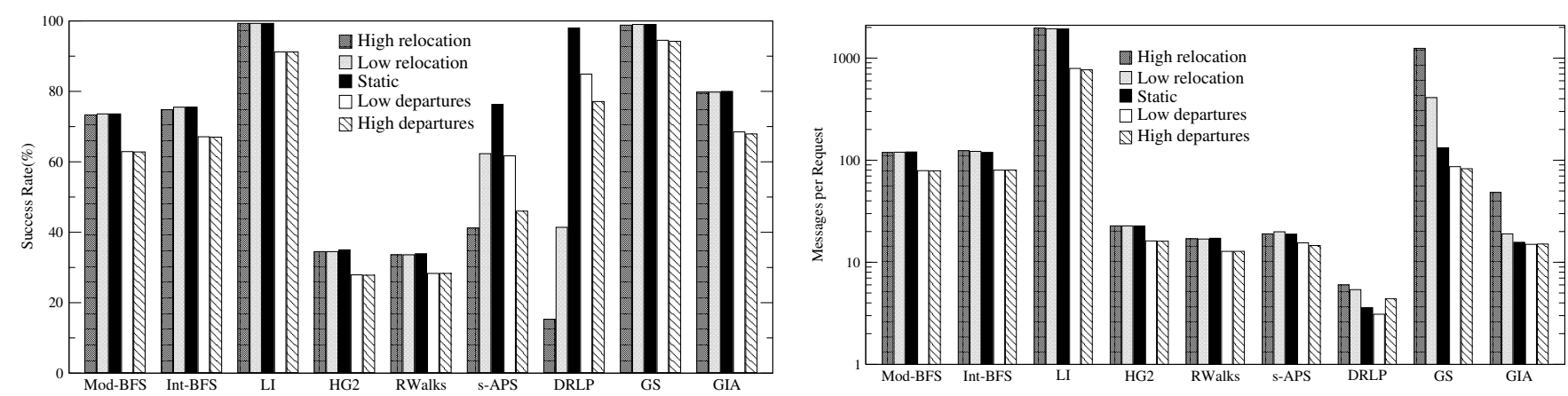

Fig. 1. Accuracy and message production for the methods using a 10,000-node random graph with average degree $d=4$

consumption is quite low (about 15 messages), while its performance is hardly affected by the dynamic operations. $H G 2$ behaves similarly, with the exception of producing about 5 more messages per search. In general, these algorithms exhibit poor performance and appear very robust to increased network variability. This is reasonable, as walkers are randomly directed with no regard to topology or previous results.

The $s$-APS method achieves a success rate of over $75 \%$ in the static run, a number that drops by around $30 \%$ in the highly dynamic settings, but only around $12 \%$ in the two less dynamic ones. The metric that is reasonably affected is the number of discovered objects, which are almost cut to a third (from 2 to 0.63 ). This happens because it takes some time for the learning feature to adapt to the new topology and paths to discovered objects frequently "disappear". On the other hand, it manages to keep its messages almost as low as Random Walks'. The scheme is equally affected by relocations and departures/arrivals, since walkers are directed towards specific locations which are altered by both types of events. Nevertheless, it exhibits a good overall performance compared to the non-BFS related schemes, without indexing other peers' repositories.

The DRLP algorithm exhibits some interesting characteristics. First, its message production is very low (less than 6 messages per request). Our simulations count the direct contact of a node (both for DRLP and $G S$ ) as one message, although a link between them might not exist in the overlay. Dynamic behavior causes the stored addresses to become more frequently "stale", thus the initial flooding is performed more often. This is the reason for the decrease in its accuracy from $99 \%$ in the static case to $77 \%$ and $15 \%$ in the highly dynamic ones. DRLP produces the same amount of messages for its initial search with Modified-BFS, so it needs many successful requests to amortize this initial cost. The number of objects it discovers is very small, ranging from 1.4 to 0.2 . If $D R L P$ is forced to use flooding many times, then the number of hits increases. If it is successful and produces few messages, then it only finds one replica per request. Despite this, we notice that it proves very bandwidth-efficient and flooding is scarcely used. This is due to the fact that, with many nodes making requests, most of them obtain a pointer for every object after a while. So, even if some node initiates a flood, most of its neighbors will only forward to one other node. This scheme seems ideal for relatively static environments and large workloads, with the exception that the number of hits will be very close to one. Another important observation is that DRLP is affected far more by object relocation than by node departures. This is reasonable if we consider that 


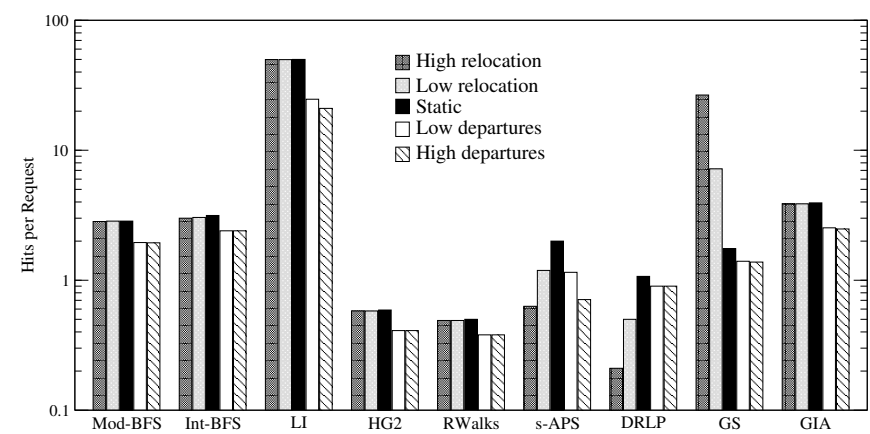

Fig. 2. Hits per query for the first graph

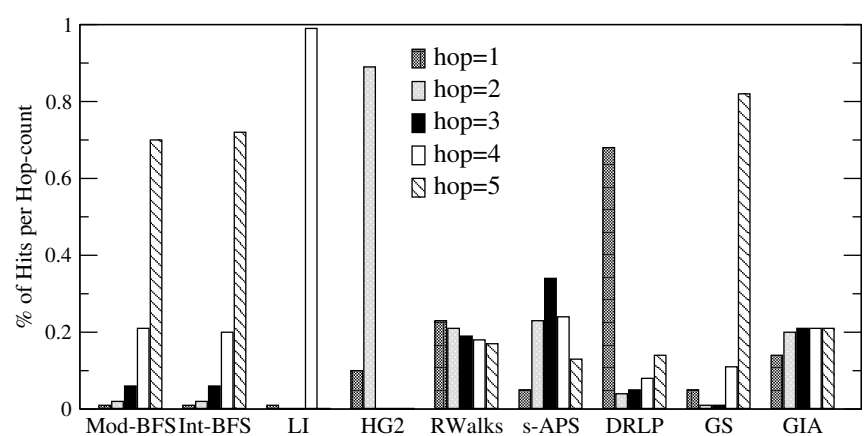

Fig. 3. \% Hits vs. Hop-distance for the second graph

with departures there still exist nodes with a valid pointer to an object, whereas object relocation may make many pointers become stale at once.

The $L I$ scheme proves the most productive in terms of discovered locations and the most costly in message production. It produces one order of magnitude more messages than the other BFS-related methods but also discovers about 10-20 times more objects, taking advantage of its index scheme. Its performance is only affected by the dynamic joins and leaves, with a decrease of more than $50 \%$ in located objects. The cost of the index updates, even under the more dynamic settings, is negligible compared to the cost of a search (at most $2 \%$ over the total number of messages). On the other hand, this cost is considerable for nodes that stay idle (and possibly alter their local repositories), since it induces traffic without any search involved.

$G S$ shows very high accuracy, since it can always fall back to the flooding scheme. Nevertheless, when peers do not have shortcuts or when these fail (this happens mostly when objects get relocated), message consumption increases dramatically. On the other hand, similarly to DRLP, the more flood searches are performed, the more objects are discovered. Shortcuts are mostly used in the static and dynamic arrival/departure modes, since 5 shortcuts proved sufficient for at least one of them to provide an answer most of the times.

Finally, GIA manages to perform as well as Mod/Int-BFS but being more bandwidth-efficient. The combination of one hop indexing and biased walkers achieves a good, robust performance at relatively low cost. Only in the high relocation setting we notice a considerable increase $(200 \%)$ in the average message consumption since peers have to refresh their indices frequently.

\section{Results on more dense graphs}

In the next set of simulations we use a random graph with an average degree $d=10$ to compare the 9 methods over two different environments: A static one, and one where both object relocation and peer departures occur about 600 times per run. DRLP and Int/Mod-BFS forward to 4 neighbors at each step, while $k=12$ for s-APS, Random Walks, HG2, GIA. All other parameters remain the same. The results for the static case are shown in Table I. We also report the percentage of messages per search that are duplicates and the average distance of the hits in overlay hops. 
TABLE I

COMPARISON ON A 10,000-NODE RANDOM GRAPH WITH DEGREE $d=10$

\begin{tabular}{|l||c|c|c|c|c|c|c|c|c|}
\hline Metric & Mod-BFS & Int-BFS & LI & HG2 & RWALKS & s-APS & DRLP & GS & GIA \\
\hline \hline Success(\%) & 98.8 & 99.8 & 100 & 70.2 & 53.4 & 91.7 & 100 & 100 & 97.0 \\
\hline Mesg & 875.0 & 1233.4 & 39710.1 & 108.7 & 43.6 & 43.0 & 8.0 & 2344.2 & 35.0 \\
\hline DupI Mesg(\%) & 10.3 & 0.4 & 18.7 & 8.3 & 0.2 & 0.1 & 1.8 & 17.8 & 0.9 \\
\hline Hits & 20.2 & 32.6 & 300.0 & 2.9 & 1.2 & 6.1 & 1.4 & 18.9 & 9.5 \\
\hline Hit Distance & 4.58 & 4.61 & 3.99 & 1.88 & 2.78 & 3.16 & 1.90 & 4.60 & 3.06 \\
\hline
\end{tabular}

Blind forwarding causes a large amount of messages to be dropped. Informed methods with no direct indices perform much better ( $s$-APS, Int-BFS wasting only $0.1 \%, 0.4 \%$ of their messages). Flood-based schemes also exibit large hop distances for their hits.

All algorithms produce a larger number of messages per request in the new graph, taking advantage of the larger number of connections. DRLP still manages to average less than 10 messages per request. Random Walks and $s$-APS roughly double their hit discovery, and increase their accuracy. On the other hand, Int/Mod-BFS produce 10 times more messages. $H G 2$ performs in between, producing about 5 times more messages. $L I$ increases its bandwidth production by more than an order of magnitude. Updates now play an even lesser role, since its search bandwidth consumption overshadows their effect. GS's performance increases similarly to $L I$ 's since they use the same underlying mechanism. Finally, GIA exhibits a very good performance again, having low message consumption and increased accuracy/hits.

Figure 3 depicts the percentage of hits discovered at various distances (1 through $T T L$ ) by the methods in the static setting. This is an important metric that shows how many objects each method locates with few or more messages. Flood-based schemes discover the vast majority of the objects $T T L$ hops away, since the available nodes increase exponentially with distance. This is the case for LI and Int/Mod-BFS. $L I$ always locates about $99 \%$ of its objects 4 hops away and the rest only 1 hop away from the requesters (since only nodes at these two depths process the queries). HG2 discovers about $90 \%$ of the objects with its flooding phase (2 hops away). Random Walks discovers almost the same number of objects per distance, since the query forwarding is done randomly. GIA also uses walkers and exhibits the same characteristic, but this is not due to random forwarding, but due to the random graph model and replication distribution in the overlay. DRLP finds almost $70 \%$ of its hits using its indices (which also explains why its hit average is close to one). The rest are discovered through the flooding phase and therefore their number increases as the distance increases for the same reason as before. $s$-APS ${ }^{1}$ displays a symmectic curve. After a certain distance, possible paths become too many and the accuracy of the indices drops. Finally, we notice that $G S$ only discovers about $5 \%$ of its hits using the shortcuts, whereas in the smaller graph the respective number was $50 \%$. This can be explained by the fact that the flooding scheme now finds 2 orders of magniture more objects than in the previous graph, while shortcuts still find one.

Figure 4 shows how object popularity affects the methods' accuracy and message production in the dynamic environment. Popularity decreases as we move to the right along the $\mathrm{x}$-axis. The first data point

\footnotetext{
${ }^{1}$ not the w-APS version that favors the discovery of nearby objects
} 


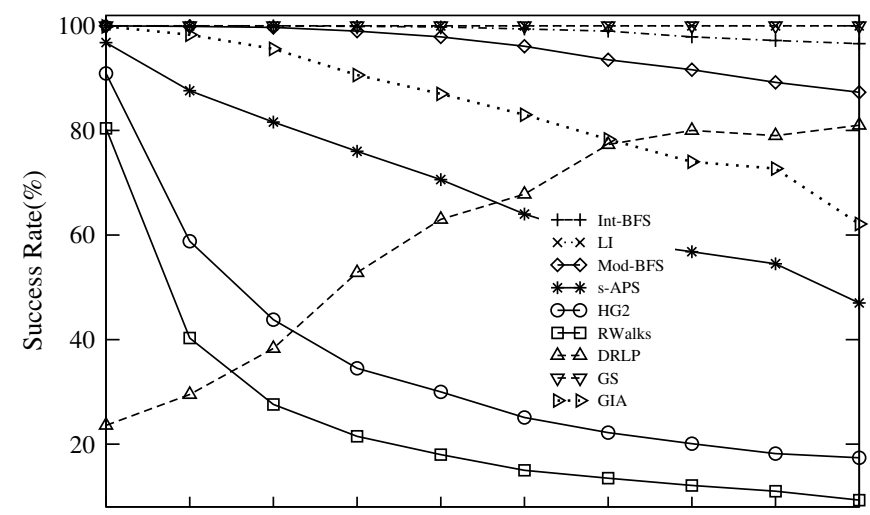

Object Popularity

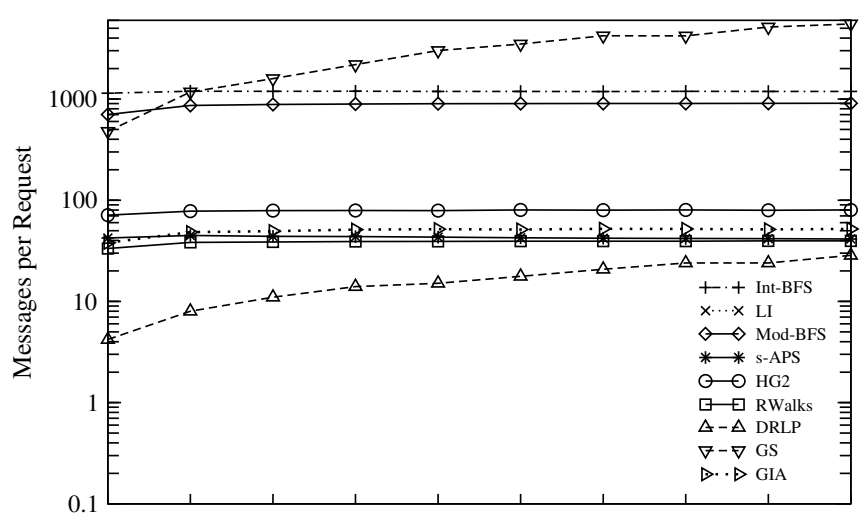

Object Popularity

Fig. 4. Accuracy and message production vs. object popularity in the dynamic setting

represents the accuracy/messages of the methods for the top-10\%, the second for objects ranked between 11-20\%, etc. This is an important comparison, because different applications or users target objects of various popularity.

The three BFS-related methods together with $G S$ exhibit very high accuracy, with Mod-BFS showing a noticeable decrease only for the least popular items. Random Walks, HG2, s-APS and GIA show decreasing accuracy as popularity drops, with GIA and $s$-APS clearly performing better. DRLP performs very poorly for the very popular documents (about 20\%), but its accuracy increases as popularity drops. This can be explained by the fact that less popular objects receive considerably fewer queries. Therefore, object relocations and node departures which affect the algorithm happen less frequently during requests for such objects. All algorithms - except DRLP and GS - waste roughly the same amount of messages per request for each popularity group. DRLP and $G S$ increase their consumption with popularity for the sole reason that the cost of the initial floods is now amortized over a smaller number of requests. In our experiments we finally noticed that all algorithms (except DRLP and GIA that deploy full flooding) discover a decreasing number of objects as popularity drops, exactly because this means there exist fewer objects to be located.

In the dynamic environment, we also measure the percentage of messages per request sent due to index updates (for relevant methods only). We found that Int-BFS requires $11 \%(=131 \mathrm{mesg}$ ) of its messages for index updates. The respective numbers for $L I, G I A$ and $s$-APS are 14.4\%(=2968 mesg), 31.7\%(=14 mesg) and $18.5 \%(=8$ mesg). Although GIA and $s-A P S$ appear to require a larger portion of updates, they are much more bandwidth-efficent than the other methods in absolute performance.

Our previous simulations depicted the relative performance characteristics of the nine algorithms. To some extent, that sort of comparison was not direct either because of the different nature of the methods or because of the single choice of the various parameters. Since it is impossible to directly compare the methods for the same parameter values (e.g., $k, T T L$ ), we select simulations using a more dense 10,000node random graph $(d=20)$, where the algorithms had similar performance in one of two important metrics: Messages and hits per query. These results were obtained by experimenting on various values 
TABLE II

COMPARISON ON A 10,000-NODE RANDOM GRAPH WITH DEGREE $d=20$

\begin{tabular}{|c|c||c|c|c|c|c|c|c|c|}
\hline \multicolumn{2}{|c|}{ Comparison Metric } & Mod-BFS & Int-BFS & HG2 & RWALKS & s-APS & DRLP & GS & GIA \\
\hline \hline \multirow{4}{*}{ Messages } & Success(\%) & 63.6 & 67.6 & 63.5 & 62.2 & 93.4 & 100 & 90.8 & 99.9 \\
\cline { 2 - 10 } & Mesg & $\mathbf{7 3 . 4}$ & $\mathbf{8 3 . 0}$ & $\mathbf{7 7 . 0}$ & $\mathbf{7 2 . 5}$ & $\mathbf{7 0 . 6}$ & $\mathbf{7 9 . 2}$ & $\mathbf{7 7 . 0}$ & $\mathbf{7 0 . 0}$ \\
\cline { 2 - 10 } & Hits & 1.9 & 2.3 & 2.1 & 2.0 & 10.7 & 5.3 & 1.12 & 14.9 \\
\hline \hline \multirow{3}{*}{ Hits } & Success(\%) & 75.8 & 77.0 & 71.9 & 75.0 & 80.2 & 100.0 & 100.0 & 92.2 \\
\cline { 2 - 10 } & Mesg & 134.4 & 117.1 & 115.1 & 125.2 & 31.4 & 43.0 & 356.5 & 32.1 \\
\cline { 2 - 10 } & Hits & $\mathbf{3 . 5}$ & $\mathbf{3 . 2}$ & $\mathbf{3 . 1}$ & $\mathbf{3 . 2}$ & $\mathbf{3 . 8}$ & $\mathbf{3 . 4}$ & $\mathbf{3 . 6}$ & $\mathbf{3 . 8}$ \\
\hline
\end{tabular}

for $k, T T L$, number of neighbors to forward and number of requester nodes. The results are presented in Table II and the comparison metric is typed in boldface. $L I$ is omitted from this table because its large message and hit production could not be matched by the other methods.

For similar message consumption, first GIA, then $s$-APS discover the most objects (followed by DRLP with about 10 extra messages per search). These three methods also prove extremely accurate, while the rest of the schemes (either flood-based or random) do not perform well. For similar hits per search, again $G I A$ and $s$-APS stand out above DRLP, which wastes few more messages but is perfectly accurate. From the rest of the methods, only $G S$ is $100 \%$ successful, but exhibits the highest message consuption.

\section{Increased number of objects}

In the previous settings, we simulated an environment where peers had multiple requests for certain objects. Imagine a network of sensors which collect information that changes with time. Sensors may also relocate or start collecting new data. Our previous model was mainly tailored for such a system, where peers want specific objects at various times (more than once) but they are not guaranteed to find them at the same place. The small number of objects (with a wide range of replication ratios) together with the varying workload and network dynamics best enables us to observe the effect of popularity, dynamic behavior and forwarding scheme. We now consider a more general situation, with a large number of objects $(20,000)$ and peers making random requests. This could be an example of a $\mathrm{P} 2 \mathrm{P}$ search engine application, with users having their own preferences (changing with time). Table III presents our comparison using two graphs, our original 10,000-node $(d=4)$ random graph and a 10,000-node $(d=4.4)$ power-law (PLAW) graph. For larger graphs (simulations up to 50,000 nodes), results are qualitatively similar.

Compared to the previous results, we clearly notice a small performance degradation, which is natural if we consider than now more queries are made for sparsely located objects, while flooding is used more by some of the methods. Nevertheless, first DRLP, followed by $s-A P S$ and GIA achieve numbers closest to the original ones. With the power-law topology, although the average out-degree is the same as with the random graph, various neighborhoods differ substantially, since there are few nodes with very high connectivity. GIA clearly takes advantage of this and discovers a number of objects comparable to $L I$, as it routes queries through the high connectivity areas. Another observation is that pure flood-based schemes 
TABLE III

COMPARISON OF THE NINE METHODS WITH A 20,000-OBJECT POOL

\begin{tabular}{|c|c||c|c|c|c|c|c|c|c|c|}
\hline \multicolumn{2}{|l|}{ Graph } & Mod-BFS & Int-BFS & LI & HG2 & RWALKS & $s$-APS & DRLP & GS & GIA \\
\hline \hline \multirow{3}{*}{ RANDOM } & Success(\%) & 68.4 & 69.7 & 89.9 & 30.7 & 29.8 & 75.2 & 99.0 & 89.2 & 74.4 \\
\cline { 2 - 11 } & Mesg & 118.8 & 115.4 & 1511.6 & 24.9 & 18.6 & 24.1 & 7.1 & 563.5 & 18.3 \\
\cline { 2 - 11 } & Hits & 2.3 & 2.4 & 37.7 & 0.5 & 0.4 & 2.2 & 1.2 & 5.0 & 3.2 \\
\hline \hline \multirow{3}{*}{ PLAW } & Success(\%) & 56.8 & 62.3 & 93.3 & 76.7 & 22.9 & 75.7 & 98.3 & 88.4 & 94.1 \\
\cline { 2 - 10 } & Mesg & 73.3 & 82.0 & 1473.0 & 750.3 & 13.1 & 15.1 & 5.0 & 355.9 & 40.7 \\
\cline { 2 - 10 } & Hits & 1.53 & 1.85 & 86.1 & 17.7 & 0.32 & 1.9 & 1.2 & 3.0 & 55.9 \\
\hline
\end{tabular}

also discover substantially more objects (again compared to the random graph simulation with 20,000 objects). HG2 achieves more than 10 times more hits with a $150 \%$ increase in accuracy, using 30 times more messages. $L I$ doubles its hits without any message increase. The rest of the schemes perform very similarly to the previous simulation.

\section{CONCLUSiOns}

This paper presents a description of current search techniques for unstructured P2P networks, along with a quantitative comparison through simulation. Our analyses focus on the performance metrics of search accuracy, bandwidth consumption, discovered objects and behavior under dynamic operations.

The specifics of the problem play a big role in choosing the right method. Each scheme has its own goals and it is important that these goals match the application's. Important parameters that could influence our decision include how dynamic the system is, what is its primary purpose (e.g., fast object discovery, many hits, bandwidth-efficient and accurate, easy deployment, combinations of them), what is the underlying topology, the expected workload, etc. We offer some general-purpose observations based on our analysis and simulations, hoping they will prove useful in evaluating the plethora of different schemes.

1) Blind forwarding is not adequate for both high performance and low cost.

2) Keeping direct pointers to more peers (e.g., DRLP, GS) is very efficient in relatively static environments

3) The index semantics play an important role. Direct location information is effective but sensitive to changes and more demanding (becomes obsolete if a failure/relocation occurs, requires update messages). Indirect information (e.g., success rates in $s$-APS, Int-BFS or connectivity/capacity in GIA) is much more robust but less accurate

4) Indexing other peers' repositories can prove very useful but must be carefully applied, since it requires updates to keep the indices up-to-date. Even when these updates are piggybacked, they still require bandwidth

5) Adaptation is sometimes desirable. Peers that have a prolonged stay in the network or route/answer/make many requests should enhance their knowledge with time. GS, s-APS and Int-BFS learn from the system queries and the more they participate, the more they improve. 
6) Experience has taught that, in many cases, the simple protocols are the preferred ones. The simplicity of the mechanisms behind flooding or random walks make them powerful and easy to implement. They can be used either by themselves or in combination with other schemes to improve their performance

Finally, we summarize our specific observations: $L I$ shows excessive bandwidth consumption even in sparse graphs, similar to GS's searches for unseen objects. A choice between Mod-BFS and Int-BFS must evaluate the trade-off between an increased bandwidth/hit production and simplicity on a welldefined context. Other blind methods (e.g., Random Walks, HG2) are simple and can reduce the amount of messages, but generally fail to perform satisfactorily and adapt to different workloads. Conversely, most informed methods achieve great results but have to cope with index maintenance. DRLP and $s$-APS require no costly updates. The former performs best in relatively static environments, deteriorating when the network is very dynamic. The latter uses its adaptive scheme to achieve a good all-around performance at low cost, but performs best with larger workloads. Finally, GIA proved a very good all-around solution, combining different ideas from other schemes. The question here relates to the overhead and effectiveness of the overlay adaptation algorithm that it proposes.

\section{REFERENCES}

[1] Clay Shirky, "What Is P2P ... And What Isn't," OpenP2P.com, 2000.

[2] "http://www.napster.com.," Napster website.

[3] "http://www.gnutella.com," Gnutella website.

[4] “http://www.kazaa.com,” Kazaa website.

[5] “http://www.jxta.org," Project JXTA.

[6] "http://www.microsoft.com/net," Microsoft .NET.

[7] "The impact of file sharing on service provider networks. An Industry White Paper, Sandvine Inc.," .

[8] "http://www.openp2p.com.," openP2P website.

[9] “http://www.peer-to-peerwg.org/.," Peer-to-Peer working group.

[10] D. Milojicic, V. Kalogeraki, R. Lukose, K. Nagaraja, J. Pruyne, B. Richard, S. Rollins, and Z. Xu, "Peer-to-Peer Computing," Tech. Rep. HPL-2002-57, HP, 2002.

[11] S. Saroiu, P. Gummadi, and S. Gribble, "A measurement study of peer-to-peer file sharing systems," Tech. Rep. UW-CSE-01-06-02, Un. of Washington, 2001.

[12] J. Chu, K. Labonte, and B. Levine, “Availability and Locality Measurements of Peer-to-Peer File Systems,” in SPIE, 2002.

[13] S. Sen and J. Wang, "Analyzing peer-to-peer traffic across large networks," in SIGCOMM Internet Measurement Workshop, 2002.

[14] S. Saroiu, K. Gummadi, R. Dunn, S. Gribble, and H. Levy, "An Analysis of Internet Content Delivery Systems," in OSDI, 2002.

[15] V. Kalogeraki, D. Gunopulos, and D. Zeinalipour-Yazti, “A Local Search Mechanism for Peer-to-Peer Networks," in CIKM, 2002.

[16] C. Lv, P. Cao, E. Cohen, K. Li, and S. Shenker, "Search and Replication in Unstructured Peer-to-Peer Networks," in ICS, 2002.

[17] D. Tsoumakos and N. Roussopoulos, "Adaptive Probabilistic Search for Peer-to-Peer Networks," in 3rd IEEE Intl Conference on P2P Computing, 2003.

[18] S. Daswani and A. Fisk, "Gnutella UDP Extension for Scalable Searches (GUESS) v0.1," .

[19] A. Crespo and H. Garcia-Molina, "Routing Indices for Peer-to-Peer Systems," in ICDCS, July 2002.

[20] M. Stokes, "Gnutella2 Specifications Part One: http://www.gnutella2.com/gnutella2_search.htm," .

[21] K. Sripanidkulchai, B. Maggs, and H. Zhang, "Efficient Content Location Using Interest-Based Locality in Peer-to-Peer Systems," in INFOCOM, 2003.

[22] Y. Chawathe, S. Ratnasamy, L. Breslau, N. Lanham, and S. Shenker, "Making Gnutella-like P2P Systems Scalable," in SIGCOMM, 2003.

[23] S. Rhea and J. Kubiatowicz, "Probabilistic Location and Routing," in INFOCOM, 2002.

[24] B. Yang and H. Garcia-Molina, "Improving Search in Peer-to-Peer Networks," in ICDCS, 2002.

[25] A. Singla and C. Rohrs, "Ultrapeers: Another Step Towards Gnutella Scalability," .

[26] D. Menascé and L. Kanchanapalli, "Probabilistic Scalable P2P Resource Location Services," SIGMETRICS Perf. Eval. Review, 2002.

[27] P. Ganesan, Q. Sun, and H. Garcia-Molina, "YAPPERS: A peer-to-peer lookup service over arbitrary topology," in INFOCOM, 2003.

[28] E. Cohen, A. Fiat, and H. Kaplan, "Associative search in peer to peer networks: Harnessing latent semantics," in INFOCOM, 2003.

[29] E. Zegura, K. Calvert, and S. Bhattacharjee, "How to model an internetwork," in Infocom, 1996. 
[30] C. Jin, Q. Chen, and S. Jamin, "Inet: Internet Topology Generator. Technical Report CSE-TR443-00, Department of EECS, University of Michigan," 2000.

[31] L. Adamic, R. Lukose, A. Puniyani, and B. Huberman, “Search in power-law networks," In press, Phys. Rev. E, 2001. 